\title{
ARTICLE
}

\section{The vagus nerve mediates the suppressing effects of peripherally administered oxytocin on methamphetamine self-administration and seeking in rats}

\author{
Nicholas A. Everett (iD) ${ }^{1}$, Anita J. Turner (iD) ${ }^{1}$, Priscila A. Costa (iD) ${ }^{1}$, Sarah J. Baracz ${ }^{1}$ and Jennifer L. Cornish ${ }^{1}$
}

The neuropeptide oxytocin has emerged as a promising pharmacotherapy for methamphetamine (METH) addiction, and clinical trials of intranasal oxytocin are underway. However, there is debate as to how peripherally administered oxytocin alters brain signalling to modulate addiction processes. Interestingly, there is evidence for functional interactions between peripheral oxytocin administration and the vagus nerve. Therefore, this study investigated whether the effects of peripherally administered oxytocin require vagal signalling to reduce METH self-administration and reinstatement of METH-seeking behaviours. Male and female Sprague-Dawley rats underwent surgery for jugular catheterisation and either subdiaphragmatic vagotomy (SDV) or a sham operation. Rats were trained to self-administer METH, and the effect of peripherally administered oxytocin on METH intake was assessed. Rats then underwent extinction, and effects of oxytocin were assessed on cue- and METH-induced reinstatement of METH-seeking. Oxytocin treatment robustly attenuated METH intake in both sexes, and SDV entirely prevented the suppressant effect of oxytocin $(0.3 \mathrm{mg} / \mathrm{kg})$ on METH intake, and partially prevented the effects of $1 \mathrm{mg} / \mathrm{kg}$ oxytocin in both sexes. After extinction, SDV decreased the suppressing effects of oxytocin on cue- and METH-primed reinstatement in males, but not females. SDV was functionally confirmed by measuring food intake following administration of the vagal dependent peptide, cholecyostokin-8. Our data suggest that vagus nerve signalling is required for the inhibitory effects of peripherally administered oxytocin on METH self-administration and reinstatement, and that this vagal dependency is partially mediated by sex and drug withdrawal. This study has implications for the use of oxytocin as a therapy for METH use disorder for both sexes.

Neuropsychopharmacology (2021) 46:297-304; https://doi.org/10.1038/s41386-020-0719-7

\section{INTRODUCTION}

Abuse of the addictive psychostimulant methamphetamine (METH) is associated with numerous psychosocial and physical risks [1]. Current psychosocial interventions for METH use disorder are not widely available and are minimally effective [2], and despite significant advances in understanding the neurobiology of METH addiction, no effective pharmacotherapies have been developed $[3,4]$. As the pharmacological agents based on these advances progress through clinical trials and continue to produce largely negative findings [5], there is an urgent need to develop new pharmacotherapies.

The neuropeptide oxytocin is a promising novel therapy for METH addiction [6-10]. Preclinical studies have shown that peripherally administered oxytocin can suppress METH selfadministration [11], and relapse-like behaviour elicited by METHcues [12-14] or by METH re-exposure [15-17]. When administered chronically during abstinence from $\mathrm{METH}$, oxytocin also protects against cue- and METH-induced relapse in male and female rats [18]. Clinical research also indicates efficacy of intranasally delivered oxytocin in reducing alcohol and marijuana cravings [19-21]. A synthesis of these preclinical and clinical findings has laid the groundwork for the first clinical trial using intranasal oxytocin in METH-dependent users [22] (NCT02881177). However, intranasal oxytocin treatment has not always been effective (see
[23] for review), which raises concerns as to whether intranasal administration in humans will recapitulate the effects of peripheral administration in rodents.

Peripheral administration of oxytocin potently reduces drugseeking behaviours, however, it is unclear how this large peptide accesses the brain following peripheral dosing to modulate neural circuits which control addiction processes [24]. Indeed, as little as $0.002-0.005 \%$ of subcutaneously or intranasally administered oxytocin can be measured in the brain $[23,25]$, while supraphysiologic concentrations of circulating oxytocin are measured following treatment with these peripheral doses [26]. As oxytocin receptors are expressed widely throughout the body [26], it is possible that peripherally administered oxytocin may signal to the brain via a peripherally mediated mechanism.

The vagus nerve bidirectionally conveys information between the brain and the peripheral organs, with up to $80 \%$ of afferent fibres signalling to the brain [27]. The primary recipient of these vagal afferents is the nucleus of the solitary tract (NTS), which has noradrenergic projections to various brain regions involved in addiction [28], as well as projections to the paraventricular (PVN) and supraoptic nuclei of the hypothalamus which appose oxytocin-secreting neurons [29]. This ascending vagal pathway is therefore well positioned to regulate the function of the endogenous oxytocin system, as stimulation of vagal nerves

${ }^{1}$ Centre for Emotional Health, Department of Psychology, Macquarie University, Marsfield, NSW, Australia

Correspondence: Nicholas A. Everett (nicholas.everett@sydney.edu.au)

Received: 5 January 2020 Revised: 11 May 2020 Accepted: 14 May 2020

Published online: 25 May 2020 
immediately elevated oxytocin levels in the peripheral bloodstream [30] and increased cFos expression, a marker of neuronal activity in the NTS and PVN [31]. Importantly, abdominal vagal fibres express oxytocin receptors [32], as well as vasopressin V1 A receptors [33], which oxytocin also acts on to reduce addictive behaviours [15], and are excited by oxytocin in ex vivo preparations [32]. Lastly, peripheral administration with oxytocin increases activity of PVN oxytocin neurons, which is prevented by subdiaphragmatic vagotomy [34]. Together, these studies provide a framework in which the vagus nerve acts as a gateway for peripherally administered oxytocin to signal to the brain to regulate behaviour.

Stimulation of the vagus nerve is being investigated as a therapy for a variety of psychiatric conditions [35], which oxytocin also has efficacy for treating, including depression [36, 37], anxiety $[38,39]$, and post-traumatic stress disorder $[40,41]$. Further, vagus nerve stimulation (VNS) has preclinical efficacy for reducing drugseeking behaviours. Specifically, chronic electrical VNS during daily extinction sessions from heroin or cocaine self-administration reduced relapse-like behaviours, and modulated activity in cortico-limbic structures involved in addiction [42,43]. Collectively, these studies indicate that peripherally administered oxytocin can stimulate the vagus nerve, and that VNS is able to modulate neural pathways involved in psychiatric conditions, which oxytocin may also ameliorate. However, it is unknown whether the inhibitory effects of peripherally administered oxytocin on METH intake or reinstatement require vagal signalling. Therefore, elucidation of the relationship between exogenous oxytocin, vagal signalling, and addiction is an important step towards effective clinical translation of oxytocin as a pharmacotherapy.

Here we investigated whether the inhibitory effects of peripherally administered oxytocin on METH self-administration and reinstatement require vagal signalling, by surgically resecting the subdiaphragmatic vagus nerve prior to volitional METH intake, reinstatement, and oxytocin treatment. Further, as preclinical evidence suggests that female rats display higher METH intake and reinstatement [44], and as the efficacy of oxytocin for reducing $\mathrm{METH}$ self-administration and reinstatement may be more potent in females [45], we investigated this hypothesised interaction of oxytocin and the vagus nerve in both sexes.

\section{METHODS AND MATERIALS}

\section{Subjects}

Sprague Dawley rats $(n=20 /$ sex) were housed in groups of 3-4 under a $12 \mathrm{~h}$ light-cycle (lights on 08:00) in open top cages $(64 \times$ $40 \times 22 \mathrm{~cm}$ ) with paper bedding, plastic tunnel, and wooden sticks. Standard chow and water were available ad libitum until experimental procedures began. Procedures were approved by the Macquarie University Animal Ethics Committee.

\section{Liquid diet}

Two days prior to surgery, solid rat chow was removed from home cages, and was replaced with a nutritionally complete liquid diet (Teklad Diet 09215) made in $90 \%$ home-cage water and $10 \%$ sweetened condensed milk for palatability. This is essential for the survival of vagotomised animals. Our observations are that subdiaphragmatic vagotomy (SDV) results in extreme gastric stasis, stomach distension, and precipitous weight loss without a liquid diet.

\section{Subdiaphragmatic vagotomy and intravenous catheter} implantation surgeries

Rats were anaesthestised with isoflurane and were randomly assigned to receive either sham surgery, or SDV. Sham rats had their bilateral subdiaphragmatic vagi isolated but not damaged, while SDV rats had a section of each nerve from the diaphragm to the stomach cut [46]. All rats were then implanted with a jugular vein catheter externalised to their midscapular region. Rats recovered for 7-10 days, and their catheters were flushed daily with heparinized cephazolin in saline. Detailed surgical procedures are described in the Supplementary.

\section{Methamphetamine self-administration training}

Rats were trained to self-administer METH $(0.1 \mathrm{mg} / \mathrm{kg} / 0.05 \mathrm{ml}$ infusion) during 14 daily fixed ratio 1 sessions ( $2 \mathrm{~h} /$ day). Selfadministration chambers were equipped with infra-red beams for locomotor tracking, two illuminated nose-pokes ('active' and 'inactive'), a house-light, and infusion pump. Active nose-pokes triggered the infusion pump and illuminated the nose-poke for $3 \mathrm{~s}$. A 20-s time-out was signalled by dimming of the house-light. Inactive nose-pokes had no consequences.

\section{Tests for the interaction of SDV and oxytocin on} methamphetamine self-administration

The effect of oxytocin $(0.3,1.0 \mathrm{mg} / \mathrm{kg}$; ChinaPeptide) or vehicle ( $0.9 \%$ saline) was tested on METH self-administration. Treatments were delivered in the home cage via intraperitoneal (IP) injection $30 \mathrm{~min}$ prior to the session. Treatments were counterbalanced across 4 test days, according to a Latin square design, with at least two self-administration days between each test.

\section{Extinction}

Rats then underwent behavioural extinction sessions for 14 days (1 h/day), during which nose-pokes had no consequences. Rats received IP saline immediately prior to the final two extinction sessions.

Tests for the interaction of SDV and oxytocin on cue-induced and methamphetamine-primed reinstatement

Following extinction, rats underwent two cue-induced reinstatement tests in the presence of METH-cues. The $1 \mathrm{~h}$ sessions began with a single non-contingent cue presentation ( $30 \mathrm{~s}$ into session). Thereafter, active nose-pokes produced contingent presentation of the METH-cue. Thirty minutes prior to these tests, rats received saline or oxytocin $(0.3 \mathrm{mg} / \mathrm{kg} \mathrm{IP})$. As repeated cue-induced reinstatement testing would result in extinction of the operant behaviour, only a single oxytocin dose was tested compared with vehicle. The lower dose was chosen due to its vagal-dependent effects on METH self-administration. Rats then underwent three METH-primed reinstatement tests, in which rats received saline or oxytocin $(0.3,1.0 \mathrm{mg} / \mathrm{kg} \mathrm{IP)} 30 \mathrm{~min}$ prior to a METH injection $(1 \mathrm{mg} / \mathrm{kg} \mathrm{IP})$, and then were placed immediately into the chamber under extinction conditions. Each reinstatement test was separated by $2-3$ extinction days. Rats did not proceed to the next test day until they reached extinction criteria ( $<10$ active pokes). Treatments were counterbalanced within each reinstatement type.

\section{Functional test of vagotomy}

For the week following reinstatement tests, food was removed from home cages ( $16 \mathrm{~h}$ deprivation from 10 p.m.-2 p.m. [47]), and rats were trained to consume their food in a metabolic chamber $(1 \mathrm{~h} /$ day at $2-3 \mathrm{pm})$. Food was returned to their home cage for another 7 hours. Once consumption was stable across 2 days, rats were tested for their intake following treatment with saline or CCK-8 (4 $\mu \mathrm{g} / \mathrm{kg}$ IP; Abcam, ab120209) $30 \mathrm{~min}$ prior to being placed in the chamber. Food consumed after $1 \mathrm{~h}$ was recorded, and effects of CCK-8 on intake is expressed in text as percent reduction of intake compared with the day prior (percentage reduction of intake $=-100$ (intake day prior - intake following CCK-8)/intake day prior).

\section{Statistical analysis}

We used $3 \times 2 \times 2$ factorial analyses of variance including the within-subjects factor of oxytocin Dose $(0,0.3,1 \mathrm{mg} / \mathrm{kg})$, and the 
- Sham Males * SDV Males

(a)

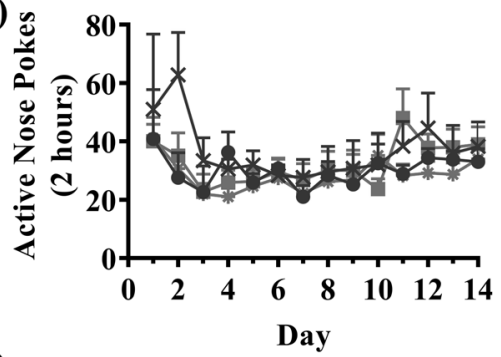

(c)

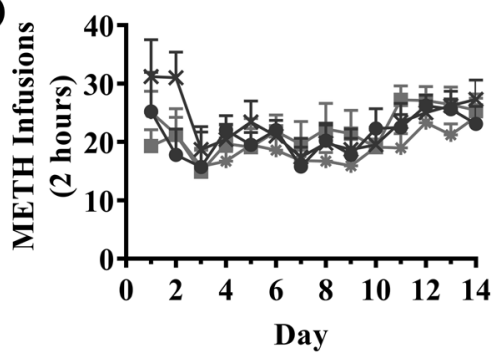

(b)

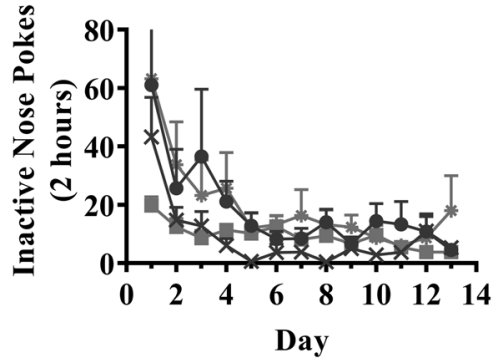

(d)

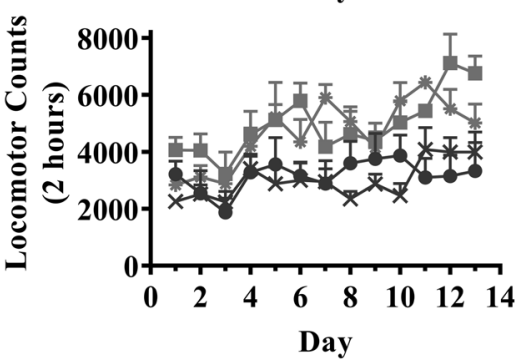

Fig. 1 Acquisition of methamphetamine self-administration. a active nose pokes; $\mathbf{b}$ inactive nose pokes; $\mathbf{c}$ METH infusions; $\mathbf{d}$ locomotor counts over 14 consecutive days of intravenous METH self-administration between sham operated and vagotomised (SDV) male and female rats. Data are mean +SEM. $n=10$ for male sham, 10 for male SDV, 8 for female sham, and 10 for female SDV.

between-subjects factors of Sex (Male, Female) and Surgery (Sham, SDV), using SPSS (Version 25, General Linear Model Procedure). If there was a main effect or interaction of Sex, then males and females were analysed in separate $3 \times 2$ models (Dose $\times$ Surgery) for self-administration and METH-primed reinstatement datasets, or a $2 \times 2$ model for cue-induced reinstatement, otherwise they were analysed together within one $3 \times 2 \times 2$ model (Dose $\times$ Surgery $\times$ Sex). If a significant interaction was found, these were followed up by $2 \times 2$ interaction tests comparing each dose of oxytocin to vehicle, and between surgery groups. Significant effects crucial for data interpretation are depicted in figures, with the remainder in Supplementary Results. These post-hoc comparisons were adjusted using Bonferroni's correction for multiple comparisons using SPSS. For selfadministration data, the analysis was performed on METH infusions earned and locomotor counts. For reinstatement data, analysis was performed on active nose pokes and locomotor counts. The first 30-min of sessions were analysed, as the effects of oxytocin dissipate after this time (Supplementary Fig. 2).

\section{RESULTS}

Excluded animals

Two female sham rats lost catheter patency during self-administration, so were excluded. During extinction, one male SDV rat was euthanized due to poor health, so their data were included in the self-administration but not the reinstatement datasets. Final sample sizes were 10 for male sham, 9-10 for male SDV, 8 for female sham, and 10 for female SDV.

Acquisition of methamphetamine self-administration

All rats acquired METH self-administration ( $>10$ infusions/session; Fig. 1). Total METH intake did not differ based on a sex $\times$ surgery interaction $(F(1,34)=0.024, p=0.899)$, sex $(F(1,34)=0.059, p=$ $0.811)$ or surgery $(F(1,34)=1.005, p=0.271)$.

Vagotomy attenuated oxytocin inhibition of methamphetamine self-administration in both sexes

For METH infusions earned, there was no significant main effect of Sex $(F(1,34)=1.691, p=0.202)$, interaction of Sex $\times$ Dose $(F(2,68)=0.980, p=0.381)$, Sex $\times$ Surgery $(F(1,34)=0.311, p=$
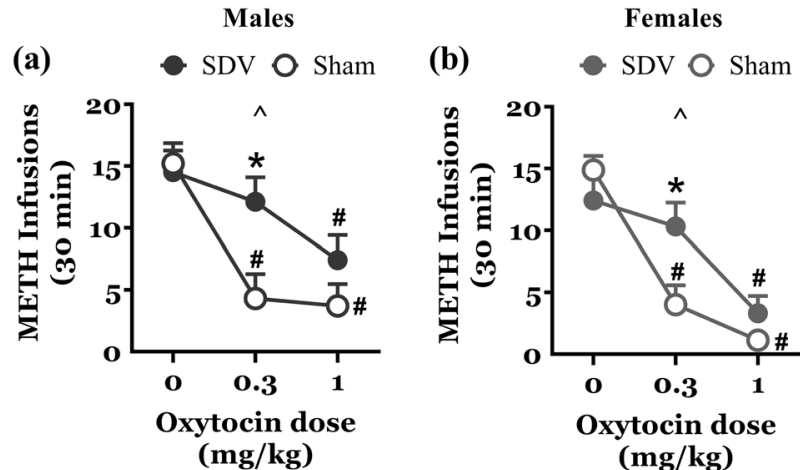

Fig. 2 The effects of IP oxytocin treatment on self-administered methamphetamine in Sham and SDV rats. Self-administered methamphetamine infusions in Sham or SDV rats following $0,0.3$ or $1.0 \mathrm{mg} / \mathrm{kg}$ oxytocin a males and $\mathbf{b}$ females. \#p<0.05 vs $0 \mathrm{mg} / \mathrm{kg}$ dose. $\wedge p<0.05$ interaction of oxytocin dose $\times$ surgery group with sexes collapsed. ${ }^{*} p<0.05$ vs respective Sham control group data point. As no sex effects were detected in this dataset, males, and females were analysed in the same model. Data are mean + SEM. $n=10$ for male sham, 10 for male SDV, 8 for female sham, and 10 for female SDV.

$0.581)$, or Sex $\times$ Surgery $\times$ Dose $(F(2,68)=0.003, p=0.997)$. Therefore, males and females were analysed together, although they are graphed separately in Fig. 2 to allow visualisation of sex-related effects. With males and females combined, there was a significant Surgery $\times$ Dose interaction $(F(2,68)=11.227, p<0.001)$, as well as a significant main effect of Dose $(F(2,68)=66.129, p<0.001)$, but not Surgery $(F(1,34)=3.805, p=0.059)$. To better understand the Surgery $\times$ Dose interaction, simple $2 \times 2$ interactions were analysed between surgery levels, and within pairs of doses. This revealed significant Surgery $\times$ Dose interactions when comparing 0 vs $0.3 \mathrm{mg} / \mathrm{kg}(F(1,34)=6.136, p=0.018)$ and 0 vs $1 \mathrm{mg} / \mathrm{kg}$ $(F(1,34)=5.671, \quad p=0.023)$, whereby both doses of oxytocin suppressed METH intake less in SDV than in sham rats. There was also an interaction of Surgery $\times$ Dose when comparing 0.3 vs $1 \mathrm{mg} / \mathrm{kg}(F(1,34)=4.497, p=0.041)$, indicating that SDV may have attenuated the inhibitory effect of oxytocin on METH intake more 


\section{Cue Induced Reinstatement}
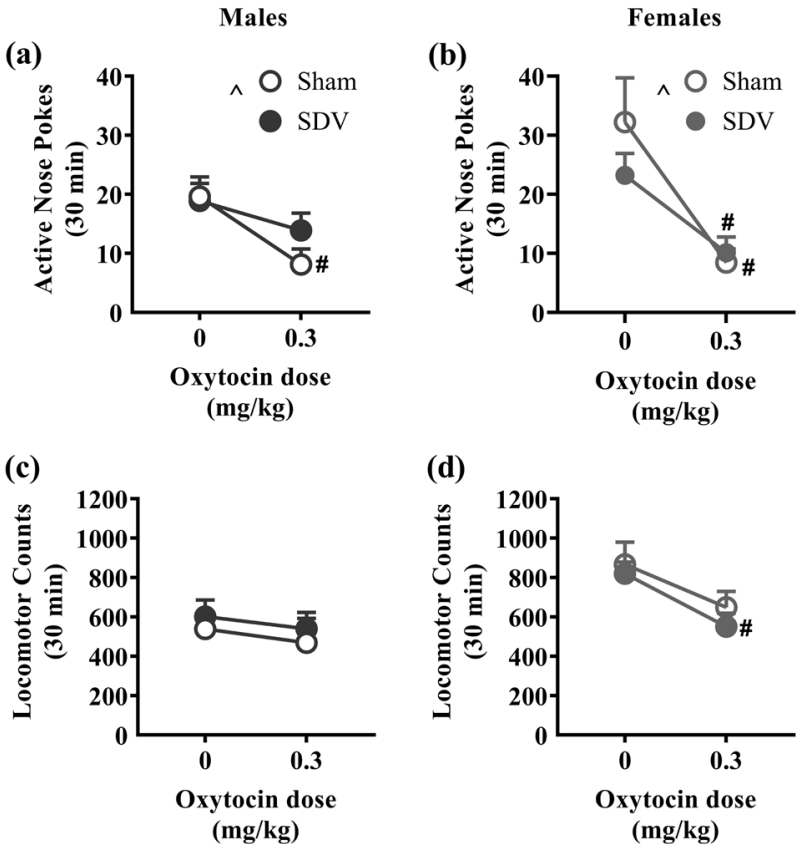

Fig. 3 The effects of IP oxytocin treatment on cue-induced reinstatement to methamphetamine-seeking behaviours in Sham and SDV rats. Cue-induced reinstatement of active nose pokes (a and b), and locomotor counts (c and d), for males (a, c) and females (b, d) respectively. $\wedge p<0.05$ interaction of oxytocin dose $\times$ surgery group with sexes collapsed. \#p $<0.05$ vs $0 \mathrm{mg} / \mathrm{kg}$ dose. ${ }^{*} p<0.05$ vs respective Sham control group data point. Data are mean + SEM. $n=10$ for male sham, 9 for male SDV, 8 for female sham, and 10 for female SDV.

so at 0.3 than at $1 \mathrm{mg} / \mathrm{kg}$. Post-hoc tests comparing METH infusions between pairs of dose, sex, and surgery are reported in Supplementary Tables 1 and 2, and are depicted in Fig. 2. Briefly, these tests indicate that compared with $0 \mathrm{mg} / \mathrm{kg}, 1 \mathrm{mg} / \mathrm{kg}$ oxytocin reduced METH infusions in both Sham and SDV male and females (all $p<0.004$ ), while $0.3 \mathrm{mg} / \mathrm{kg}$ only reduced METH infusions in Sham male and females $(p=0.001$ and $p<0.001$ respectively), but not in SDV male and females ( $p=0.147$ and $p=$ 0.135 respectively).

Extinction of methamphetamine self-administration

Rats decreased their active nose-pokes and locomotor activity over the 14 days of extinction (for all groups, Day 1 vs 14: $p<0.05$ ), and all rats met standard criteria of $<10$ active nose-pokes before proceeding to reinstatement tests (Supplementary Fig. 1).

Vagotomy attenuated oxytocin inhibition of cue-induced reinstatement in males but not females

For active nose pokes made during cue-induced reinstatement, there was no significant main effect of Sex $(F(1,33)=1.297, p=$ $0.263)$, Sex $\times$ Surgery $(F(1,33)=1.115, p=0.299)$, or Sex $\times$ Surgery $\times$ Dose $(F(1,33)=0.975, p=0.331)$. However, there was a significant interaction of Surgery $\times$ Dose $(F(1,33)=5.711, p=$ 0.023 ) whereby, with sexes combined, the inhibitory effects of oxytocin on cue-induced reinstatement were attenuated in SDV compared with Sham rats. Additionally, there was a significant interaction of Sex $\times$ Dose $(F(1,33)=7.480, p=0.010)$. When analysed only within Sham rats, the Sex $\times$ Dose interaction was maintained $(F(1,16)=4.882, p=0.042)$, indicating that oxytocin was more potent in reducing cue-induced reinstatement in females compared with males. Therefore, males and females were further analysed in separate $2 \times 2$ models (Surgery $\times$ Dose). For
Methamphetamine Primed Reinstatement (a)

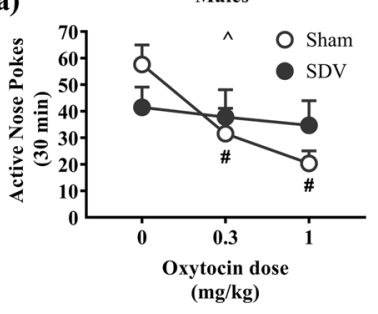

(c)

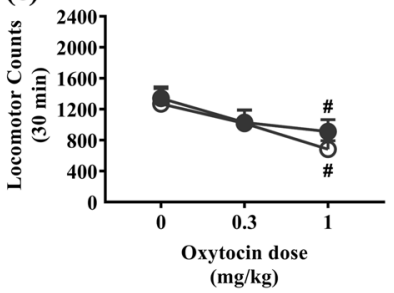

(b)

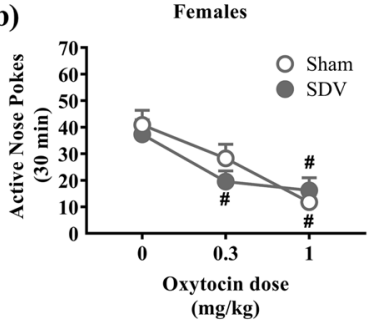

(d)

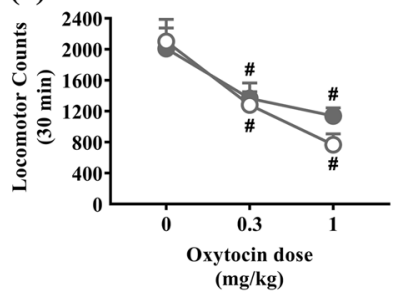

Fig. 4 The effects of IP oxytocin treatment on methamphetamineprimed reinstatement to drug-seeking behaviours in Sham and SDV rats. Methamphetamine-primed reinstatement of active nose pokes $(\mathbf{a}, \mathbf{b})$, and locomotor counts (c, $\mathbf{d})$, for males $(\mathbf{a}, \mathbf{c})$ and females $(\mathbf{b}, \mathbf{d})$ respectively. $\wedge p<0.05$ interaction of oxytocin dose $\times$ surgery group for males only. $\# p<0.05$ vs $0 \mathrm{mg} / \mathrm{kg}$ dose. ${ }^{*} p<0.05$ vs respective Sham control group data point. Data are mean + SEM. $n=10$ for male sham, 9 for male SDV, 8 for female sham, and 10 for female SDV.

males, there was a significant main effect of Dose $(F(1,17)=$ $10.319, p=0.005)$, indicating that $0.3 \mathrm{mg} / \mathrm{kg}$ oxytocin significantly reduced cue-induced reinstatement, compared with $0 \mathrm{mg} / \mathrm{kg}$. There was no main effect of Surgery $(F(1,17)=0.566, p=0.462)$, and no interaction of Surgery $\times$ Dose $(F(1,17)=1.601, p=0.223)$. Post-hoc pairwise tests indicate that compared with $0 \mathrm{mg} / \mathrm{kg}$, $0.3 \mathrm{mg} / \mathrm{kg}$ oxytocin reduced cue-induced reinstatement in Sham males $(p=0.011)$, but not in SDV males $(p=0.211)$. For females, there was a significant main effect of Dose $(F(1,16)=28.458, p<$ 0.001 ), indicating that $0.3 \mathrm{mg} / \mathrm{kg}$ oxytocin significantly reduced cue-induced reinstatement, compared with $0 \mathrm{mg} / \mathrm{kg}$. There was no main effect of Surgery $(F(1,16)=0.576, p=0.459)$, and no significant interaction of Dose $\times$ Surgery $(F(1,16)=3.980, p=$ $0.063)$. Post-hoc pairwise tests indicate that compared with $0 \mathrm{mg} / \mathrm{kg}, 0.3 \mathrm{mg} / \mathrm{kg}$ oxytocin reduced cue-induced reinstatement in both Sham females $(p=0.011)$, and SDV females $(p<0.001)$. Simple contrasts comparing locomotor activity between individual levels of dose, sex, and surgery are depicted in Fig. 3, and in Supplementary Tables 3-6.

Vagotomy attenuated oxytocin inhibition of methamphetamineprimed reinstatement in males but not females

For active nose pokes made during METH-primed reinstatement (Fig. 4), there was a significant main effect of $\operatorname{Sex}(F(1,33)=4.658$, $p=0.038)$, but no significant interactions of Sex $\times$ Surgery $\times$ Dose $(F(1,33)=1.915, p=0.156)$ or Sex $\times$ Dose $(F(1,33)=0.101, p=$ $0.904)$. As there was a main effect of Sex, and as three levels of dose were assessed, males and females were further analysed in separate $2 \times 2$ models (Surgery $\times$ Dose). For males, this revealed a significant Surgery $\times$ Dose interaction when comparing 0 vs $1 \mathrm{mg} /$ $\mathrm{kg}(F(1,17)=8.529, p=0.010)$, but not 0 vs $0.3 \mathrm{mg} / \mathrm{kg}(F(1,17)=$ $2.844, p=0.110)$, indicating that $1 \mathrm{mg} / \mathrm{kg}$ oxytocin reduced METHprimed reinstatement more so in sham than SDV males. Post-hoc pairwise tests indicate that compared with $0 \mathrm{mg} / \mathrm{kg}, 0.3 \mathrm{mg} / \mathrm{kg}$ oxytocin reduced METH-primed reinstatement of reinstatement in Sham males $(p=0.022)$, but not in SDV males $(p=0.704)$, and $1 \mathrm{mg} / \mathrm{kg}$ also reduced reinstatement in Sham males $(p=0.003)$, but not in SDV males $(p=0.169)$. For females, there were no 


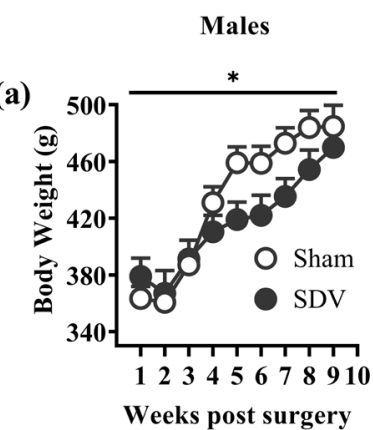

(b)
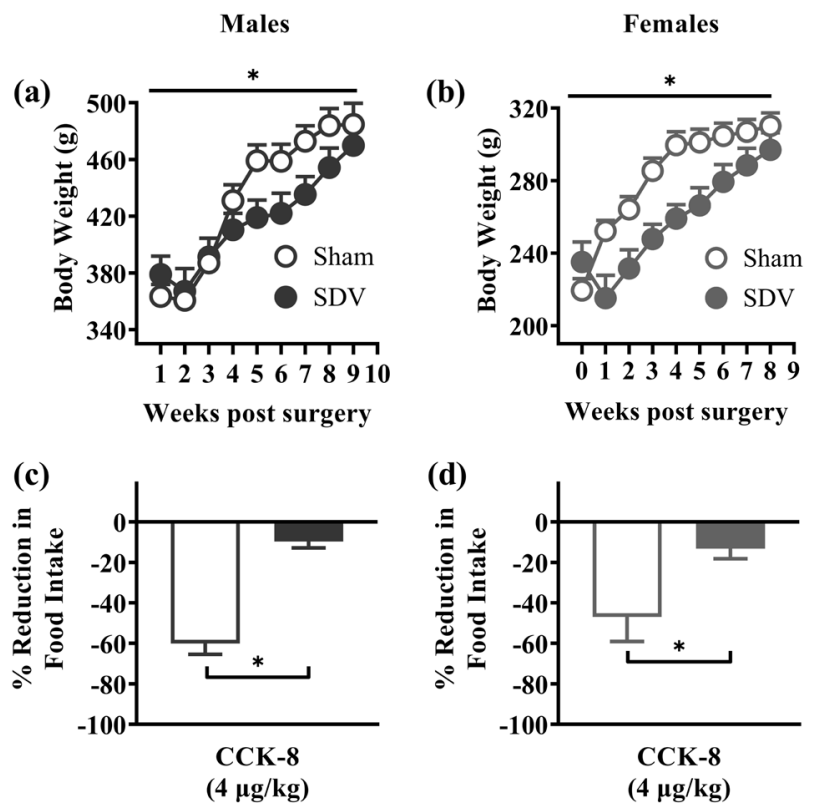

Fig. 5 Body weight, and functional validation of successful subdiaphragmatic vagotomy by CCK-8 inhibition of feeding in Sham and SDV rats. Body weight over 10 weeks for Sham and SDV males (a) and females (b). Percent reduction of intake of liquid rodent diet following IP injection of CCK-8 $(4 \mathrm{ug} / \mathrm{kg})$ in Sham and SDV males (c) and females (d). $p<0.05$ between Sham and SDV rats. Data are mean + SEM. $n=10$ for male sham, 9 for male SDV, 8 for female sham, and 10 for female SDV.

significant Surgery $\times$ Dose interactions when comparing 0 vs $0.3 \mathrm{mg} / \mathrm{kg}(F(1,16)=0.531, p=0.477)$ or 0 vs $1 \mathrm{mg} / \mathrm{kg}(F(1,16)=$ $0.760, p=0.396)$, indicating that the suppressant effects of either dose of oxytocin on METH-primed reinstatement was unaffected by SDV. In further support of this, post-hoc pairwise tests indicate that compared with $0 \mathrm{mg} / \mathrm{kg}, 0.3 \mathrm{mg} / \mathrm{kg}$ oxytocin reduced METHprimed reinstatement in Sham females, although this did not reach significance $(p=0.059)$. In SDV females, $0.3 \mathrm{mg} / \mathrm{kg}$ significantly reduced METH-primed reinstatement compared with $0 \mathrm{mg} /$ $\mathrm{kg}(p=0.003)$. Compared with $0 \mathrm{mg} / \mathrm{kg}, 1 \mathrm{mg} / \mathrm{kg}$ reduced reinstatement in both Sham females $(p=0.002)$, and SDV females $(p=0.012)$. Simple contrasts comparing locomotor activity between individual levels of dose, sex, and surgery are depicted in Fig. 3, and in Supplementary Tables 7-10.

Vagotomy was functionally confirmed by cholecystokinine-8 suppression of food consumption

There was a significant main effect of surgery $(F(1,33)=6.037, p=$ 0.019; Fig. 5), whereby SDV rats had lower body weight than sham rats, and a significant interaction of Surgery $\times$ Week $(F(8,272)=$ $6.524, p<0.001)$, indicating the surgery-induced weight differences dissipated over time. There was no Sex $\times$ Surgery interaction for weight $(F(1,33)=0.099, p=0.755)$. CCK-8 treatment reduced feeding by at least $35 \%$ in all sham rats, whereas no SDV rats had a reduction of intake greater than $24 \%$. There was a significant effect of Surgery $(F(1,33)=37.902, p<0.001)$, whereby feeding was reduced in sham but not SDV rats. There was no interaction of Sex $\times$ Surgery $(F(1,33)=1.486, p=0.234)$, and no main effect of Sex $(F(1,33)=0.516, p=0.478)$, on reduction of food intake following CCK-8 treatment.

\section{DISCUSSION}

Here we present the novel finding that the inhibitory effect of peripherally administered oxytocin on METH self-administration and reinstatement depends on subdiaphragmatic vagal signalling.
Specifically, the effect of a low dose of oxytocin $(0.3 \mathrm{mg} / \mathrm{kg})$ to inhibit METH self-administration was absent in male and female rats, which had undergone subdiaphragmatic vagotomy, yet reduced $\mathrm{METH}$ intake in sham controls. Conversely, the inhibitory effect of a higher dose of oxytocin $(1 \mathrm{mg} / \mathrm{kg})$ on METH intake was only partially attenuated by vagotomy in males and females. After a period of extinction, vagotomy reduced the inhibitory effects of oxytocin treatment on cue-induced and METH-primed reinstatement to METH-seeking behaviours in males, but not females. These data provide evidence for a role of vagal signalling in the therapeutic effects of oxytocin on METH intake and METHreinstatement, and suggest that sex-dependent oxytocin-vagal mechanisms emerge following METH extinction or withdrawal.

Vagotomy attenuates the inhibitory effects of peripheral oxytocin treatment on METH self-administration and reinstatement

Oxytocin administered at $0.3 \mathrm{mg} / \mathrm{kg}$ potently reduced METH selfadministration, which was entirely prevented by SDV in both sexes. However, at the $1 \mathrm{mg} / \mathrm{kg}$ oxytocin dose, this vagal dependency was less pronounced. One explanation for this dose-dependent involvement of the vagus nerve for the effect of oxytocin is increased penetration of the blood-brain barrier at higher doses of oxytocin [48]. Recent studies in oxytocin-null mice, which cannot produce oxytocin, demonstrate that peripheral oxytocin injection results in a substantial increase in brain oxytocin levels $[49,50]$. As such, it is possible that the brain concentrations of oxytocin achieved by the low dose are insufficient to inhibit METH self-administration in SDV rats. Rather, suppression of METH intake by this low dose in shams may be achieved through stimulation of an ascending vagal pathway to the brain. In contrast, the high oxytocin dose may result in brain concentrations of oxytocin which are sufficient to inhibit METH seeking, despite the lack of vagal signalling. Both sexes were similar with regard to the vagal-dependent effects of IP oxytocin on suppressing METH-intake. These data clearly implicate the vagus nerve as a crucial target for IP oxytocin in reducing METH intake, and yet are not inconsistent with observations of behaviourally relevant blood-brain passage of peripherally administered oxytocin, particularly at higher doses.

Following extinction of drug seeking and withdrawal from $\mathrm{METH}$, the role of the vagus nerve in mediating the effects of oxytocin on METH behaviours appears to be more complex. In Sham operated males, $0.3 \mathrm{mg} / \mathrm{kg}$ oxytocin reduced cue-induced reinstatement, while $0.3 \mathrm{mg} / \mathrm{kg}$ did not reduce cue-induced reinstatement in vagotomised males. For METH-primed reinstatement, 0.3 and $1.0 \mathrm{mg} / \mathrm{kg}$ oxytocin potently reduced METH-primed reinstatement in shams males but had no effect in SDV males, which was separable from the effect of oxytocin on METH-induced locomotor activity. These results indicate that after undergoing extinction and withdrawal, the vagus nerve mediates the inhibitory effects of peripheral oxytocin treatment on METHseeking behaviours in males. In contrast, $0.3 \mathrm{mg} / \mathrm{kg}$ oxytocin in females potently reduced cue-induced reinstatement, and 0.3 and $1.0 \mathrm{mg} / \mathrm{kg}$ oxytocin reduced METH-primed reinstatement similarly in sham and SDV groups. The female reinstatement data following $0.3 \mathrm{mg} / \mathrm{kg}$ oxytocin contrast with the vagal dependent effects of $0.3 \mathrm{mg} / \mathrm{kg}$ on METH self-administration, although the apparent vagal-independent effects of $1 \mathrm{mg} / \mathrm{kg}$ oxytocin in females appear to be consistent across self-administration and METH-primed reinstatement datasets.

The divergent interactions of SDV, oxytocin, and sex between self-administration and reinstatement are challenging to interpret, due to the lack of female subjects included in oxytocin, METH, and vagal research. However, there are several possible explanations for the present sex differences. Firstly, there is evidence that expression of oxytocin receptors is altered by chronic METH selfadministration and extinction in male rodents [51, 52]. As females and males have non-identical patterns of oxytocin receptor 
expression [53] it is possible that METH- and extinction-induced plasticity of oxytocin receptors may result in an augmented sensitivity to the concentration of IP oxytocin which crosses the BBB in SDV females. Alternatively, METH self-administration causes BBB injury [54], which may result in greater penetration of IP oxytocin into the brain. Indeed, the mechanism by which oxytocin is transported into the brain has recently been identified [49], and this system may be affected by METH exposure [55]. However, the effect of METH exposure and withdrawal on oxytocin receptors or BBB integrity has mostly been studied in male subjects, so it is unclear whether there are sex-dependent effects of METH on either of these systems. Lastly, the effects of VNS in addiction models have also only been explored in males [42, 43], rendering it unknown whether VNS can achieve similar inhibitory effects on drug-seeking behaviours in females.

It is unlikely that the altered vagal dependency of oxytocin treatment observed in females following extinction could be due to regeneration of vagal nerve endings over the 3 weeks separating self-administration and reinstatement tests. Vagal regeneration can occur after $\sim 5$ months post-SDV in rats, however even after this time it is estimated that only 7-39\% of fibres regenerate [56]. Given the 3-week timespan separating our vagal-dependent and vagal-independent effects of oxytocin in females, regeneration is unlikely to explain our data. Indeed, if this effect was due to vagal regeneration, then the enduring vagal dependency of oxytocin in males would suggest profound sex differences in vagal regrowth. We are not aware of any literature to support sex-dependent regeneration of peripheral nerves to this extent. Regardless, functional vagotomy was confirmed in both sexes after reinstatement tests by administering the satiety-signalling peptide CCK-8, which inhibits feeding through subdiaphragmatic vagal signalling [57]. This test at the very least confirms that vagal afferents sensitive to CCK-8 were non-functional. As oxytocin has been shown to stimulate a similar population of nodose ganglion neurons as CCK-8 [32], we can be confident that the sex-dependent mediation of oxytocin treatment on reinstatement by SDV is not due to vagal regeneration.

Vagal-brain pathways which may enable oxytocin to reduce METH self-administration and reinstatement

Activation of the vagus nerve by peripheral oxytocin treatment produces a cascade of effects along a central projecting pathway. The first step of this pathway involves vagal innervation of the NTS. Importantly, peripheral oxytocin injection increases neuronal activity within the NTS, which is entirely prevented by SDV [32]. Noradrenergic neurons in the NTS project to widespread structures with relevance to substance abuse, including the bed nucleus of the stria terminalis [58] which can regulate dopaminergic activity [59], and to the central amygdala [60], which contributes to reinstatement of METH seeking [61]. It is possible that IP oxytocin modulates activity at these addiction-relevant structures via a vagal-NTS pathway.

Stimulation of this vagal-NTS pathway may also inhibit drugseeking behaviours through activation of the endogenous oxytocin system. Noradrenergic neurons in the NTS project to the PVN [62] and can activate oxytocin cells [63]. Indeed, lesions to the NTS markedly reduce reactivity of PVN oxytocin cells in response to a peripheral inflammatory challenge [29]. Stimulation of oxytocin neurons via a vagal-NTS-PVN pathway is further supported by recent demonstration that SDV prevents IP oxytocin from increasing activity of NTS-PVN projecting neurons, and prevents oxytocin release in the PVN [34]. Oxytocin-secreting cells in the PVN project to a variety of structures implicated in addiction. For example, stimulation of oxytocin fibres in the central amygdala causes local GABAergic inhibition [64], an effect which has been shown to reduce cue-induced reinstatement to METH seeking [61]. Therefore, IP oxytocin may stimulate endogenous release of oxytocin at sites, which govern drug seeking, via this vagal-NTS-PVN pathway.

The vagal-independent effects of the high oxytocin dose may be explained by circulating oxytocin stimulating other peripheral structures. For example, high doses of oxytocin may avoid the vagal-dependent access to this NTS-PVN pathway through stimulation of the BBB-deficient area postrema, which mediates the ability of the NTS to stimulate PVN oxytocin release [65]. Furthermore, high levels of peripherally circulating oxytocin could reach the nodose ganglion, activating these vagi cell bodies, which project to the NTS. Such a nodose ganglion-mediated effect has been shown in the transmission of peripheral immune signals to the brain [66], and may explain our findings. Alternatively, oxytocin at high doses may bypass this vagal dependency by acting upon vasopressin V1A receptors, an effect which has been shown to reduce METH-primed reinstatement [15]. Indeed, as V1A receptors are located on peripheral vagal fibres [33], and as there are substantial sex differences in the expression of V1A throughout the brain [53], the present interaction of sex, withdrawal, and vagotomy on the effects of the high dose of oxytocin may involve V1A binding.

Future directions and implications for translation of oxytocin to the clinic

It is important to note that the present data do not indicate that the vagal-dependent effect of peripherally administered oxytocin is specific to reducing METH behaviours. Indeed, others have shown a role for vagal-dependent oxytocin treatment in reducing food intake [32, 34]. Therefore, one possible hypothesis which leads from these findings is that the vagal pathway mediates the effects of administered oxytocin on reward-processing more generally. Given the ability of oxytocin to modulate dopaminergic signalling in the midbrain [67-69] and nucleus accumbens $[70,71]$, this is plausible, and may underpin the ability of oxytocin treatment to reduce drug intake, drug withdrawal, and/or drugseeking for heroin [72], nicotine [73], cocaine [74-76], alcohol $[20,21,71,77]$, and for other substances of abuse [70]. However, this is challenging to test with typical non-drug reward control experiments which utilise sucrose self-administration (e.g. [78]), as oxytocin signals satiety for carbohydrates [79], and regulates ingestive behaviour through a hypothalamic-hindbrain feeding pathway [80], which is probably not involved in the present METH behaviours. As such, we suggest that future experiments utilise a recently developed operant social self-administration model [81] to determine whether the dose-dependent pro-social effects of IP oxytocin treatment [82] are similarly mediated by the vagus nerve.

Our findings have implications for the design and interpretation of clinical studies, and for the applicability of oxytocin as a pharmacotherapy for METH-use disorders. Firstly, it is crucial that sex is included as a biological variable. Currently, the only registered trial investigating the efficacy of oxytocin on METH addiction outcomes is in treatment seeking HIV-positive males [22]. As such, it should not be presumed that the results of this trial will inform the suitability of oxytocin as a therapy for females, who according to our findings, and others [44], may be more sensitive to oxytocin treatment, and may have non-identical peripheral-to-central signalling pathways for oxytocin. Furthermore, as the vagal-dependent effects of peripherally administered oxytocin may change throughout withdrawal, oxytocin should be explored at timepoints encompassing current use, and into abstinence. Lastly, as intranasal delivery of oxytocin in the clinic gains both momentum and controversy [23], the differences between intranasal and IP administration must be considered. Unlike IP, IN oxytocin does not increase activity in the mouse NTS [83], which may preclude the therapeutic benefits of stimulating this vagal-mediated pathway. Furthermore, IP oxytocin produces substantially higher plasma levels of oxytocin than IN [84], which may contribute to the vagal-dependent effects of IP 
administration. However, as IN oxytocin does elevate plasma levels of oxytocin, it would be intriguing to investigate whether the behavioural effects of IN oxytocin are prevented by SDV. The translation of oxytocin from IP in rodents to IN in humans may depend on how important this oxytocin-stimulated vagal-brain pathway is for treating addictions in humans, and whether IN oxytocin can stimulate this pathway.

\section{CONCLUSIONS}

Here we have discovered that the well documented preclinical effects of peripherally administered oxytocin in reducing METH self-administration and reinstatement to METH-seeking behaviours is largely dependent upon the subdiaphragmatic vagus nerve. Sex differences in this vagal dependency of oxytocin's effects emerged after withdrawal, which has implications for the clinical utility of oxytocin. Lastly, the growing momentum towards intranasal delivery of oxytocin to bypass the blood-brain barrier should be considered in light of these findings, which indicate that in rats, some amount of the therapeutic effects of oxytocin for treating METH intake and reinstatement is mediated by the vagus nerve. As such, drugs or implants which target oxytocinsensitive vagal pathways may prove to be valuable therapies for METH addiction.

\section{FUNDING AND DISCLOSURE}

This research was supported by the Department of Psychology, Macquarie University. NAE and PAC were recipients of Australia Postgraduate Award scholarships. This manuscript has been posted on the preprint server bioRxiv. SJB and JLC hold a patent for a pharmacotherapy for addictions. All other authors report no financial interests or potential conflicts of interest.

\section{ACKNOWLEDGEMENTS}

We thank Justin Clarke and Ronny Eidels for their assistance with designing the surgical procedures, Christine Sutter for her all-encompassing support, and Virginia Williams, Carlie Crawford, Julie Reynolds, and the animal technicians for their daily support.

\section{AUTHOR CONTRIBUTIONS}

NAE conceived the study. NAE, SJB and JLC designed the experiments, and AJT designed the surgical procedure. NAE, AJT and JLC performed surgeries. NAE and SJB carried out post-operative care. NAE carried out self-administration experiments. NAE and PAC carried out feeding experiments. NAE conducted data analysis and wrote the manuscript. All authors critically reviewed the content and approved the final version before submission.

\section{ADDITIONAL INFORMATION}

Supplementary Information accompanies this paper at (https://doi.org/10.1038/ s41386-020-0719-7)

Publisher's note Springer Nature remains neutral with regard to jurisdictional claims in published maps and institutional affiliations.

\section{REFERENCES}

1. Darke S, Kaye S, McKETIN R, Duflou J. Major physical and psychological harms of methamphetamine use. Drug Alcohol Rev. 2008;27:253-62.

2. A meta-analytic review of psychosocial interventions for substance use disorders. Am J Psychiatry. https://ajp.psychiatryonline.org/doi/full/10.1176/appi. ajp.2007.06111851\#.

3. Forray A, Sofuoglu M. Future pharmacological treatments for substance use disorders. Br J Clin Pharmacol. 2014;77:382-400.

4. Morley KC, Cornish JL, Faingold A, Wood K, Haber PS. Pharmacotherapeutic agents in the treatment of methamphetamine dependence. Exp Opin Investig Drugs. 2017;26:563-78.
5. Ballester J, Valentine G, Sofuoglu M. Pharmacological treatments for methamphetamine addiction: current status and future directions. Exp Rev Clin Pharm. 2017;10:305-14.

6. McGregor IS, Bowen MT. Breaking the loop: oxytocin as a potential treatment for drug addiction. Horm Behav 2012;61:331-9.

7. Sarnyai Z, Kovács GL. Oxytocin in learning and addiction: from early discoveries to the present. Pharmacol Biochem Behav. 2014;119:3-9.

8. Baracz SJ, Cornish JL. The neurocircuitry involved in oxytocin modulation of methamphetamine addiction. Front Neuroendocrinol. 2016;43:1-18.

9. Lee MR, Weerts EM. Oxytocin for the treatment of drug and alcohol use disorders. Behav Pharmacol. 2016;27:640-8.

10. Bowen MT, Neumann ID. Rebalancing the addicted brain: oxytocin interference with the neural substrates of addiction. Trends Neurosci. 2017:40:691-708.

11. Carson DS, Cornish JL, Guastella AJ, Hunt GE, McGregor IS. Oxytocin decreases methamphetamine self-administration, methamphetamine hyperactivity, and relapse to methamphetamine-seeking behaviour in rats. Neuropharmacology. 2010;58:38-43.

12. Cox BM, et al. Oxytocin acts in nucleus accumbens to attenuate methamphetamine seeking and demand. Biol Psychiatry. 2017;81:949-58.

13. Everett N, Baracz S, Cornish J. Oxytocin treatment in the prelimbic cortex reduces relapse to methamphetamine-seeking and is associated with reduced activity in the rostral nucleus accumbens core. Pharmacol Biochem Behav. 2019;183:64-71.

14. Bernheim A, Leong K-C, Berini C, Reichel CM. Antagonism of $m G l u 2 / 3$ receptors in the nucleus accumbens prevents oxytocin from reducing cued methamphetamine seeking in male and female rats. Pharmacol Biochem Behav. 2017;161:13-21.

15. Everett NA, McGregor IS, Baracz SJ, Cornish JL. The role of the vasopressin V1A receptor in oxytocin modulation of methamphetamine primed reinstatement. Neuropharmacology. 2018;133:1-11.

16. Baracz SJ, Everett NA, McGregor IS, Cornish JL. Oxytocin in the nucleus accumbens core reduces reinstatement of methamphetamine-seeking behaviour in rats. Addict Biol. 2016;21:316-25.

17. Baracz SJ, Everett NA, Cornish JL. The involvement of oxytocin in the subthalamic nucleus on relapse to methamphetamine-seeking behaviour. PLOS One. 2015;10: e0136132.

18. The effect of chronic oxytocin treatment during abstinence from methamphetamine self-administration on incubation of craving, reinstatement, and anxiety. Neuropsychopharmacology. https://www.nature.com/articles/s41386019-0566-6.

19. McRae-Clark AL, Baker NL, Maria MM-S, Brady KT. Effect of oxytocin on craving and stress response in marijuana-dependent individuals: a pilot study. Psychopharmacology. 2013;228:623-31.

20. Pedersen $C A$, et al. Intranasal oxytocin blocks alcohol withdrawal in human subjects. Alcohol Clin Exp Res 2013;37:484-9.

21. Hansson AC, et al. Oxytocin reduces alcohol cue-reactivity in alcohol-dependent rats and humans. Neuropsychopharmacology. 2018;43:1235-46.

22. Stauffer CS, et al. Oxytocin-enhanced motivational interviewing group therapy for methamphetamine use disorder in men who have sex with men: study protocol for a randomized controlled trial. Trials. 2019;20:145.

23. Leng G, Ludwig M. Intranasal oxytocin: myths and delusions. Biol Psychiatry. 2016;79:243-50.

24. Mens WBJ, Witter A, Van Wimersma Greidanus TB. Penetration of neurohypophyseal hormones from plasma into cerebrospinal fluid (CSF): half-times of disappearance of these neuropeptides from CSF. Brain Res. 1983;262:143-9.

25. Born J, et al. Sniffing neuropeptides: a transnasal approach to the human brain. Nat Neurosci 2002;5:514-6.

26. Kimura $\mathrm{T}$, et al. Molecular regulation of the oxytocin receptor in peripheral organs. J. Mol. Endocrinol. 2003;109-15. https://doi.org/10.1677/jme.0.0300109.

27. Prechtl JC, Powley TL. The fiber composition of the abdominal vagus of the rat. Anat Embryol. 1990;181:101-15.

28. Delfs JM, Zhu Y, Druhan JP, Aston-Jones GS. Origin of noradrenergic afferents to the shell subregion of the nucleus accumbens: anterograde and retrograde tracttracing studies in the rat. Brain Res. 1998;806:127-40.

29. Buller K, Xu Y, Dayas C, Day T. Dorsal and ventral medullary catecholamine cell groups contribute differentially to systemic interleukin- $1 \beta$-induced hypothalamic pituitary adrenal axis responses. Neuroendocrinology. 2001;73:129-38.

30. Stock S, Uvnäs-Moberg K. Increased plasma levels of oxytocin in response to afferent electrical stimulation of the sciatic and vagal nerves and in response to touch and pinch in anaesthetized rats. Acta Physiol Scand. 1988;132:29-34.

31. Cunningham JT, Mifflin SW, Gould GG, Frazer A. Induction of c-Fos and $\Delta$ FosB Immunoreactivity in Rat Brain by Vagal Nerve Stimulation. Neuropsychopharmacology. 2008;33:1884-95.

32. Iwasaki $Y$, et al. Peripheral oxytocin activates vagal afferent neurons to suppress feeding in normal and leptin-resistant mice: a route for ameliorating hyperphagia and obesity. Am J Physiol. 2014;308:R360-R369. 
33. Gao X, et al. Presence of functional vasopressin $\mathrm{V} 1$ receptors in rat vagal afferent neurones. Neurosci Lett. 1992;145:79-82.

34. Iwasaki Y, et al. Relay of peripheral oxytocin to central oxytocin neurons via vagal afferents for regulating feeding. Biochem Biophys Res Commun. 2019;519:553-8.

35. Yuan $H$, Silberstein SD. Vagus nerve and vagus nerve stimulation, a comprehensive review: part II. Headace J Head Face Pain. 2016:56:259-66.

36. De Cagna F, et al. The role of intranasal oxytocin in anxiety and depressive disorders: a systematic review of randomized controlled trials. Clin Psychopharmacol Neurosci. 2019;17:1-11.

37. Rush $\mathrm{AJ}$, et al. Vagus nerve stimulation for treatment-resistant depression: a randomized, controlled acute phase trial. Biol Psychiatry. 2005;58:347-54.

38. Neumann ID, Slattery DA. Oxytocin in general anxiety and social fear: a translational approach. Biol Psychiatry. 2016;79:213-21.

39. George MS, et al. A pilot study of vagus nerve stimulation (VNS) for treatmentresistant anxiety disorders. Brain Stimul. 2008;1:112-21.

40. Olff M, Langeland W, Witteveen A, Denys D. A psychobiological rationale for oxytocin in the treatment of posttraumatic stress disorder. CNS Spectr 2010;15:522-30

41. Noble $\mathrm{L}$, et al. Effects of vagus nerve stimulation on extinction of conditioned fear and post-traumatic stress disorder symptoms in rats. Transl Psychiatry. 2017;7:e1217-e1217.

42. Liu $\mathrm{H}$, et al. Vagus nerve stimulation inhibits heroin-seeking behavior induced by heroin priming or heroin-associated cues in rats. Neurosci Lett. 2011;494:70-74.

43. Childs JE, DeLeon J, Nickel E, Kroener S. Vagus nerve stimulation reduces cocaine seeking and alters plasticity in the extinction network. Learn Mem. 2017;24:35-42.

44. Cox BM, Young AB, See RE, Reichel CM. Sex differences in methamphetamine seeking in rats: impact of oxytocin. Psychoneuroendocrinology. 2013;38:2343-53.

45. Dluzen DE, Liu B. Gender differences in methamphetamine use and responses: a review. Gend Med. 2008;5:24-35.

46. Mravec B, Ondicova K, Tillinger A, Pecenak J. Subdiaphragmatic vagotomy enhances stress-induced epinephrine release in rats. Auton Neurosci. 2015;190:20-25.

47. Zhang J, Ritter RC. Circulating GLP-1 and CCK- 8 reduce food intake by capsaicininsensitive, nonvagal mechanisms. Am J Physiol -Regul Integr Comp Physiol. 2012;302:R264-R273.

48. Bowen MT. Does peripherally administered oxytocin enter the brain? Compelling new evidence in a long-running debate. Pharmacol Res. 2019;146:104325.

49. Yamamoto $Y$, et al. Vascular RAGE transports oxytocin into the brain to elicit its maternal bonding behaviour in mice. Commun Biol. 2019;2:1-13.

50. Smith AS, Korgan AC, Young WS. Oxytocin delivered nasally or intraperitoneally reaches the brain and plasma of normal and oxytocin knockout mice. Pharmacol Res. 2019;146:104324.

51. Baracz, SJ, et al. Chronic methamphetamine self-administration dysregulates oxytocin plasma levels and oxytocin receptor fibre density in the nucleus accumbens core and subthalamic nucleus of the rat. J Neuroendocrinol. 2016;28. https://doi.org/10.1111/jne.12337.

52. Georgiou $P$, et al. Methamphetamine abstinence induces changes in $\mu$-opioid receptor, oxytocin and CRF systems: association with an anxiogenic phenotype. Neuropharmacology. 2016;105:520-32.

53. Smith CJW, et al. Age and sex differences in oxytocin and vasopressin V1a receptor binding densities in the rat brain: focus on the social decision-making network. Brain Struct Funct. 2017:222:981-1006.

54. Ramirez SH, et al. Methamphetamine disrupts blood-brain barrier function by induction of oxidative stress in brain endothelial cells. J Cereb Blood Flow Metab 2009;29:1933-45.

55. Treweek JB, Dickerson TJ, Janda KD. Drugs of abuse that mediate advanced glycation end product formation: a chemical link to disease pathology. Acc Chem Res 2009:42:659-69.

56. Phillips RJ, Baronowsky EA \& Powley TL. Regenerating vagal afferents reinnervate gastrointestinal tract smooth muscle of the rat. 22 .

57. Abdominal vagotomy blocks the satiety effect of cholecystokinin in the rat. Science. https://science.sciencemag.org/content/213/4511/1036?casa token= PQ8D1MeDzfEAAAAA:H6LgcaUUXRU3RgqnxVVC5ngzqDTPLsqGJDPliHojRuwDL ndXL5_RVejhFuSo4UsF4AiQyRg-x96aKho.

58. Aston-Jones G, Delfs JM, Druhan J, Zhu Y. The bed nucleus of the stria terminalis: a target site for noradrenergic actions in opiate withdrawal. Ann N Y Acad Sci USA 1999:877:486-98.

59. Jalabert M, Aston-Jones G, Herzog E, Manzoni O, Georges F. Role of the bed nucleus of the stria terminalis in the control of ventral tegmental area dopamine neurons. Prog Neuropsychopharmacol Biol Psychiatry. 2009;33:1336-46.
60. Petrov T, Krukoff TL, Jhamandas JH. Branching projections of catecholaminergic brainstem neurons to the paraventricular hypothalamic nucleus and the central nucleus of the amygdala in the rat. Brain Res. 1993;609:81-92.

61. Li X, Zeric T, Kambhampati S, Bossert JM, Shaham Y. The central amygdala nucleus is critical for incubation of methamphetamine craving. Neuropsychopharmacology. 2015;40:1297-306.

62. Cunningham ET, Sawchenko PE. Anatomical specificity of noradrenergic inputs to the paraventricular and supraoptic nuclei of the rat hypothalamus. J Comp Neurol. 1988;274:60-76.

63. Onaka T, Luckman SM, Antonijevic I, Palmer JR, Leng G. Involvement of the noradrenergic afferents from the nucleus tractus solitarii to the supraoptic nucleus in oxytocin release after peripheral cholecystokinin octapeptide in the rat. Neuroscience. 1995;66:403-12.

64. Knobloch HS, et al. Evoked axonal oxytocin release in the central amygdala attenuates fear response. Neuron. 2012;73:553-66.

65. Carter DA, Lightman SL. A role for the area postrema in mediating cholecystokinin-stimulated oxytocin secretion. Brain Res. 1987;435:327-30.

66. Hosoi T, Okuma Y, Matsuda T, Nomura Y. Novel pathway for LPS-induced afferent vagus nerve activation: possible role of nodose ganglion. Auton Neurosci. 2005;120:104-7.

67. Xiao L, Priest MF, Nasenbeny J, Lu T, Kozorovitskiy Y. Biased oxytocinergic modulation of midbrain dopamine systems. Neuron. 2017;95:368-.e5.

68. Charlet A, Grinevich V. Oxytocin mobilizes midbrain dopamine toward sociality. Neuron. 2017;95:235-7.

69. Baracz SJ, Cornish JL. Oxytocin modulates dopamine-mediated reward in the rat subthalamic nucleus. Horm Behav. 2013;63:370-5.

70. Kohli $\mathrm{S}$, et al. Oxytocin attenuates phencyclidine hyperactivity and increases social interaction and nucleus accumben dopamine release in rats. Neuropsychopharmacology. 2019;44:295-305.

71. Peters ST, Bowen MT, Bohrer K, McGregor IS, Neumann ID. Oxytocin inhibits ethanol consumption and ethanol-induced dopamine release in the nucleus accumbens. Addict Biol. 2017;22:702-11.

72. Kovács GL, Borthaiser Z, Telegdy G. Oxytocin reduces intravenous heroin selfadministration in heroin-tolerant rats. Life Sci. 1985;37:17-26.

73. Manbeck KE, Shelley D, Schmidt CE, Harris AC. Effects of oxytocin on nicotine withdrawal in rats. Pharmacol Biochem Behav. 2014;116:84-89.

74. Kohtz AS, Lin B, Smith ME, Aston-Jones G. Attenuated cocaine-seeking after oxytocin administration in male and female rats. Psychopharmacology. 2018;235:2051-63.

75. Weber RA, et al. Regionally specific effects of oxytocin on reinstatement of cocaine seeking in male and female rats. Int J Neuropsychopharmacol. 2018;21:677-86.

76. Kovàcs GL, Sarnyai Z, Babarczi E, Szabó G, Telegdy G. The role of oxytocindopamine interactions in cocaine-induced locomotor hyperactivity. Neuropharmacology. 1990;29:365-8.

77. Tunstall BJ, et al. Oxytocin blocks enhanced motivation for alcohol in alcohol dependence and blocks alcohol effects on GABAergic transmission in the central amygdala. PLOS Biol. 2019;17:e2006421.

78. Hay GL, et al. Cannabidiol treatment reduces the motivation to self-administer methamphetamine and methamphetamine-primed relapse in rats. J Psychopharmacol. 2018;32:1369-78.

79. Herisson FM, Brooks LL, Waas JR, Levine AS, Olszewski PK. Functional relationship between oxytocin and appetite for carbohydrates versus saccharin. NeuroReport. 2014;25:909-14.

80. Olszewski PK, Klockars A, Schiöth HB, Levine AS. Oxytocin as feeding inhibitor: maintaining homeostasis in consummatory behavior. Pharmacol Biochem Behav. 2010;97:47-54

81. Venniro $M$, Shaham $Y$. An operant social self-administration and choice model in rats. Nat Protoc. 2020;15:1542-59.

82. Ramos $L$, et al. Acute prosocial effects of oxytocin and vasopressin when given alone or in combination with 3,4-methylenedioxymethamphetamine in rats: involvement of the V1 A receptor. Neuropsychopharmacology. 2013; 38:2249-59.

83. Maejima $Y$, et al. Nasal oxytocin administration reduces food intake without affecting locomotor activity and glycemia with c-Fos induction in limited brain areas. Neuroendocrinology. 2015;101:35-44.

84. Neumann ID, Maloumby R, Beiderbeck DI, Lukas M, Landgraf R. Increased brain and plasma oxytocin after nasal and peripheral administration in rats and mice. Psychoneuroendocrinology. 2013;38:1985-93. 